roben Keimen in den ersten Zeiten nach dem Tode.) (Istit. di med. leg., univ., Cagliari e Parma.) Giorn. di batteriol. e immunol. Jg. 2, Nr. 6, S. 321-357. 1927.

Verff. haben 26 menschliche und 4 tierische Leichen in den ersten Zeiten nach dem Tode (von 16 Stunden bis 30 Tagen für menschliche Leichen) auf Anaerobier untersucht: Von den menschlichen, waren 13 Leichen infolge gewaltsamen Todes, 13 infolge Krankheit gestorben. Als Untersuchungsmaterial diente die Milz. -. Auf Grund ihrer Beobachtungen gelangen die Verff. zur Utberzeugung einer chronologischen Aufeinanderfolge verschiedener Arten von Anaerobier in den ersten Tagen nach dem Tode, welches wohl auf Änderungen der chemischen Zusammensetzung des Milieus beruht: dieses kann durch innerliche oder äußerliche Bedingungen (Art des Todes, Temperatur usw.) beschleunigt oder verzögert, nicht aber geändert werden. Das Aufeinanderfolgen.der Keime kann folgendermaßen schematisiert werden: Zu Anfang tritt der Fraenkelsche Bac. auf, dann die Butirici, endlich die Putrifici. Die Lebensbedingungen und die chemische Tätigkeit der Keime verursachen die verschiedenen Fäulniserscheinungen: anfangs das von den kohlehydratspaltenden, gasbildenden Butirici hervorgerufene Fäulnisemphysem, dann die Kolliquation, welche von den eiweißspaltenden, kaum gasbildenden Putrifici bedingt wird. Verff. glauben deswegen, daß das Studium des Aufeinanderfolgens der Anaerobier an Leichen keine genaueren Schlüsse auf die Zeit des Todes gestattet als die Beobachtung der von ihnen hervorgerufenen groben und allbekannten Fäulniserscheinungen.

Romanese (Parma).

Alessandrini, Giulio: Sull'importanza degli insetti nella distruzione dei cadaveri. (Über die Bedeutung der Insekten bei der Zerstörung der Leichen.) (Istit. d'ig., univ., Roma.) Ann. d'ig. Jg. 37, Nr. 8, S. 497-514. 1927.

Alessandrini studiert die oben genannte Frage an getöteten und 12 Stunden nach dem Tode in den Sommermonaten begrabenen Meerschweinchen. Die Exhumierung und die Untersuchung derselben folgte zwischen 15 und 80 Tagen nach dem Tode: der Einfluß der Art des Begrabens, der Zusammensetzung des Sarges, der Anwendung von Desinfektionsmitteln wird besonders erörtert. Verschiedene Arten von Insekten (Sp. Lucilia caesar, Cortonera stabulans, Phora aterrima, Arthomyja vicina; die Dipteren, abgesehen von einem Falle, fehlten!), wurden regelmäßig gefunden, wo die Leichen in einfachen Holzsärgen lagen und an unbedeckten Orten zwischen 15 und 56 Tagen beerdigt blieben. Bei den an bedeckten Orten begrabenen Leichen wurden dagegen keine Insekten gefunden; in diesem Falle zeigten die Leichen eine gewisse Neigung zur Vertrocknung. Die Zerstörung geht schneller da, wo Insekten dazwischen kommen: im allgemeinen, je schwerer die Verbindungsmöglichkeit zwischen Leiche und Außenwelt, desto langsamer wird die Zerstörung vollendet: am längsten also dauert es, wenn die Leiche in einem Zinksarge liegt. Wenn Desinfektionsmittel angewandt werden, bleibt die Leiche längere Zeit unverändert. Die Arbeit, welche eher hygienische als gerichtsärztliche Richtung hat, bietet doch einige interessante Anhaltspunkte bezüglich der Zeitbestimmung des Todes. (Vgl. dies. Zeitschr. 10, 683.)

Romanese (Parma).

\title{
Versicherungsrechtliche Medizin.
}

- Kramer, Franz: Neurologische Untersuchungs-Sehemata. Periphere und spinale Sensibilitätsbezirke nebst Blättern zum Eintragen von Sensibilitätsbefunden. Reizpunkte der Nerven und Muskeln. Berlin: Julius Springer 1927. 6 Abb. u. 50 Doppelformulare. RM. 4.80.

Das Heft enthält zunächst Schemata der elektrischen Reizpunkte, dann die der peripheren und weiterhin die der spinalen Sensibilitätsbezirke nach den neuesten Erfahrungen und schließlich Blätter zur Eintragung der Befunde, in denen jede Begrenzung fehlt, um eine Eintragung des erhobenen Befundes ohne jede suggestive Verfälschung und erst nachträgliche Vergleichung mit dem Schema zu ermöglichen. Diese Anordnung erscheint sehr zweckmäßig, ebenso ist das Format des Blattes ein genügend großes. Die Hefte müssen deshalb jedem; der genauere Sensibilitätsprüfungen machen will, dringend empfohlen werden.

F. Stern (Göttingen). 